\title{
Sub-critical water processing of proteins: An alternative to enzymatic digestion?
}

\author{
Thomas Powell ${ }^{1}$, Steve Bowra ${ }^{2}$ and Helen J. Cooper ${ }^{1 *}$ \\ ${ }^{1}$ School of Biosciences, University of Birmingham, Edgbaston, Birmingham B15 2TT, UK. \\ ${ }^{2}$ Phytatec (UK) Ltd., Plas Gogerddan, Aberystwyth SY23 3EB, UK
}

\section{Supplementary Information}

Supplementary Figure 1 - Photograph of SCW reaction tube

Supplementary Figure $\mathbf{2}$ - Percentage of peptide spectral matches following protein database search versus treatment conditions. $n=3$. Error bars represent one standard deviation.

Supplementary Figure 3 - Summed survey scan mass spectra obtained following LC MS/MS analysis of SCW treated hemoglobin. (a) SCW treatment of $1 \mathrm{mg} / \mathrm{ml} \mathrm{Hb}$; (b) SCW treatment of $0.1 \mathrm{mg} / \mathrm{ml} \mathrm{Hb}$; (c) SCW treatment of $0.01 \mathrm{mg} / \mathrm{ml} \mathrm{Hb}$.

Supplementary Figure 4 - Specificity of SCW hydrolysis: Plots of the \% of peptides identified against the C-terminal amino acid residue for proteins haemoglobin, BSA and $\beta$-casein under SCW treatment at $160{ }^{\circ} \mathrm{C}$ (0 mins), $160^{\circ} \mathrm{C}$ (20 mins) and $207^{\circ} \mathrm{C}$ (20 mins). $\mathrm{n}=3$. Error bars represent one standard deviation.

Supplementary Figure 5 - Summary of peptides identified following SCW hydrolysis of A) haemoglobin, B) BSA and C) $\beta$-casein under conditions $160^{\circ} \mathrm{C}$ for 0 minutes, $160^{\circ} \mathrm{C}$ for 20 minutes, $207^{\circ} \mathrm{C}$ for 20 minutes, $253^{\circ} \mathrm{C}$ for 20 minutes and $300^{\circ} \mathrm{C}$ for 20 minutes.

Supplementary Figure 6 - Amino acid sequence of $\beta$-casein. Phophorylated residues are shown in lower case.

Supplementary Figure 7 - ETD MS/MS spectra of phosphopeptides produced following SCW hydrolysis or trypsin digestion of $\beta$ casein. 


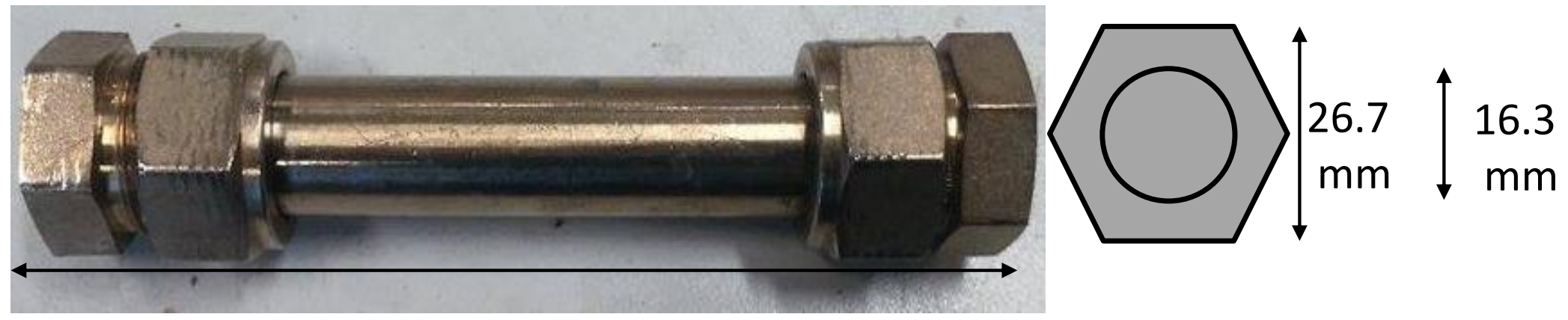

$200 \mathrm{~mm}$

SCW processing: Reaction tubes were placed in the oven pre-set to $30{ }^{\circ} \mathrm{C}$ and allowed to equilibrate for at least 10 minutes prior to increasing the temperature of the oven. The reaction temperature was monitored by use of a thermocouple attached to one of the reaction tubes. It took 6 minutes 40 seconds to reach 160 ${ }^{\circ} \mathrm{C}, 7$ minutes 40 seconds to reach $207^{\circ} \mathrm{C}, 10$ minutes 30 seconds to reach $253^{\circ} \mathrm{C}$ and 16 minutes 45 seconds to reach $300^{\circ} \mathrm{C}$. The selected residency, i.e., 0 mins and 20 mins, began after reaching the chosen temperature. 


\section{Supplementary Figure 2}

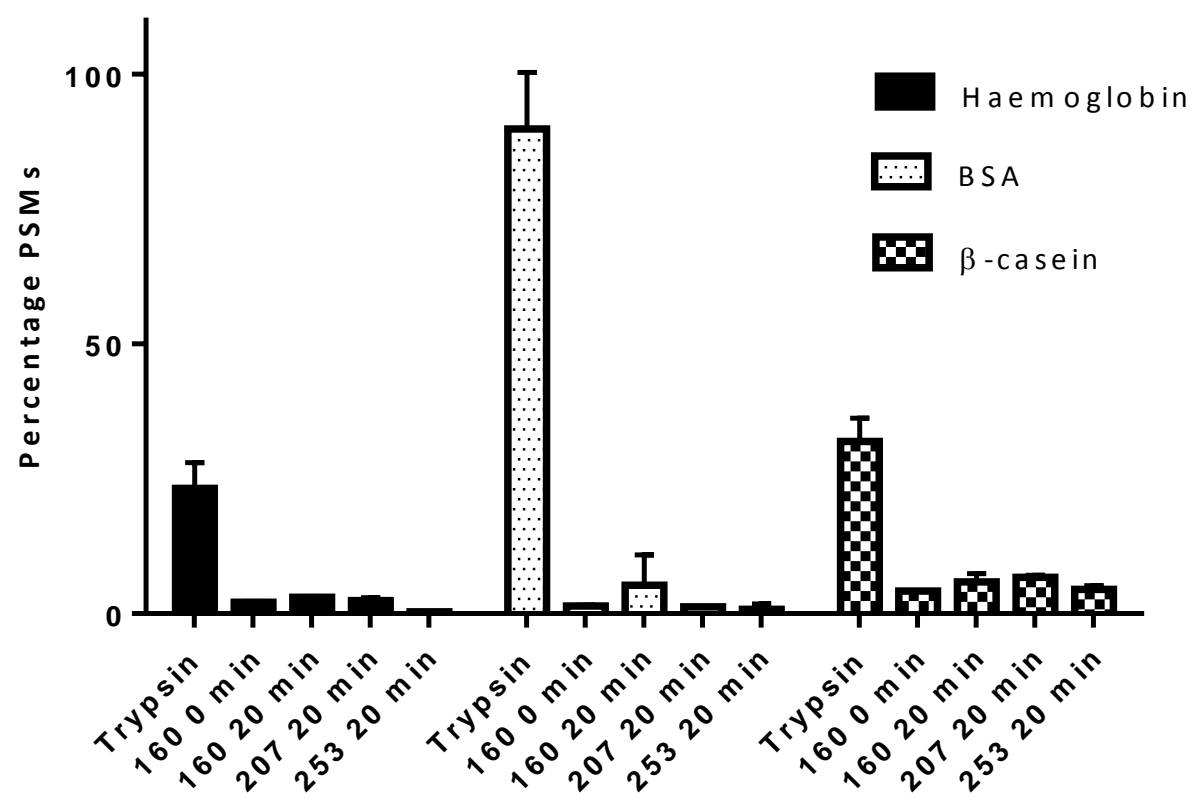


Supplementary Figure 4

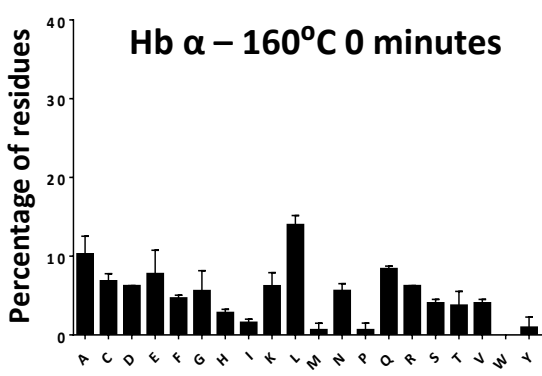

Amino acid residue

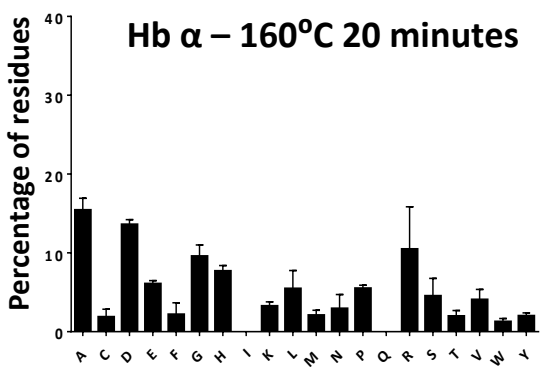

Amino acid residue

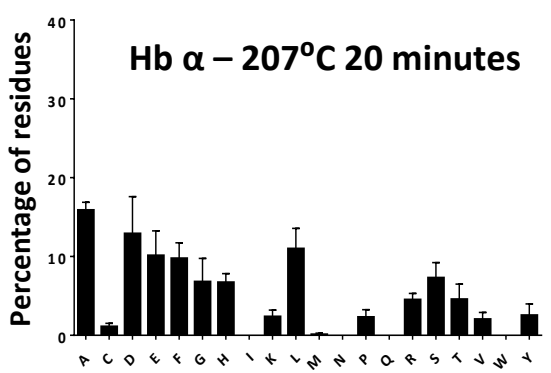

Amino acid residue

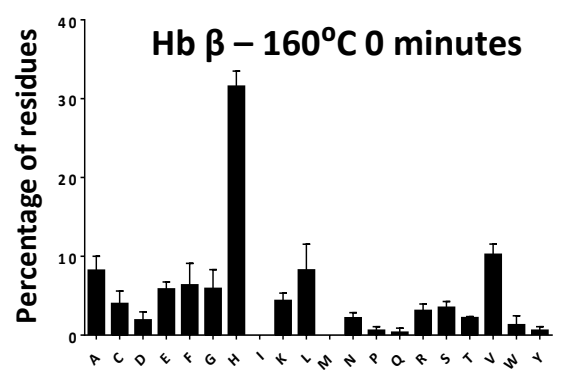

Amino acid residue

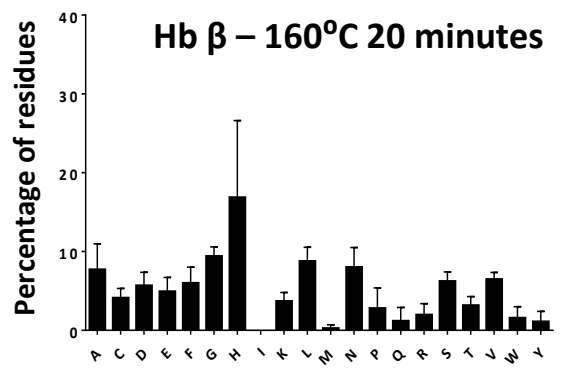

Amino acid residue

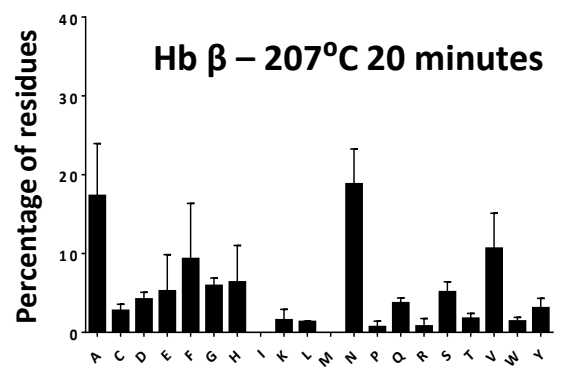

Amino acid residue

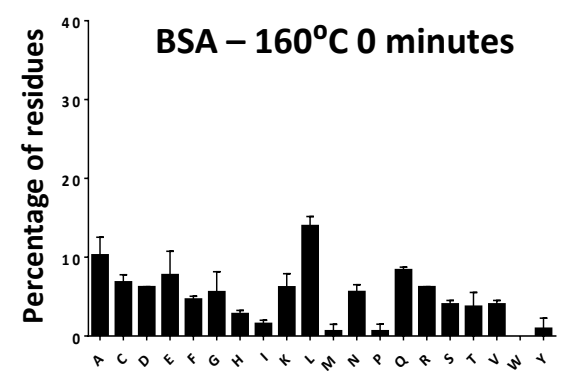

Amino acid residue

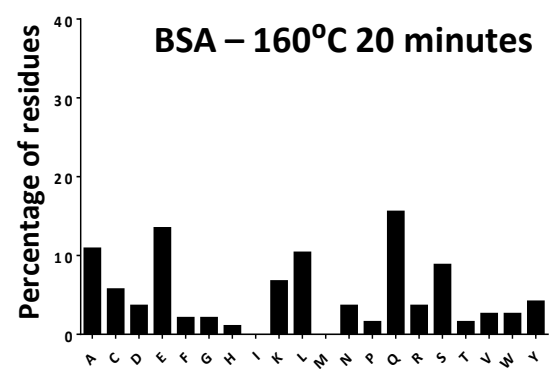

Amino acid residue

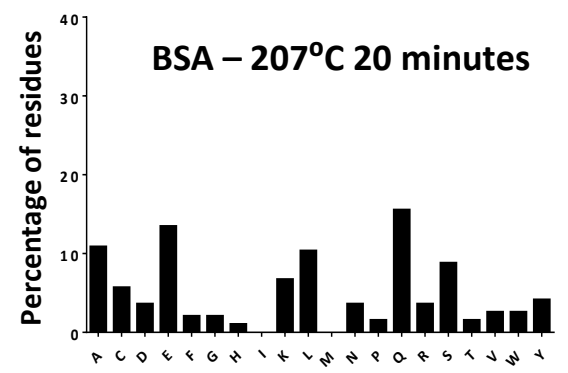

Amino acid residue

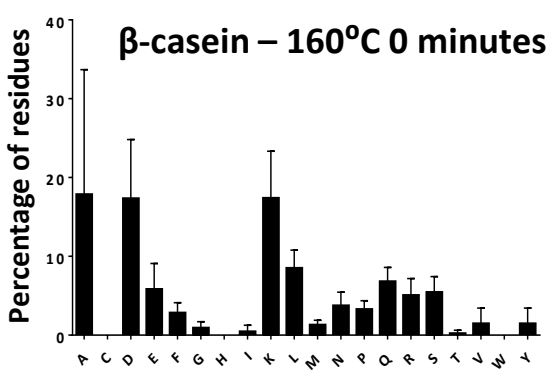

Amino acid residue

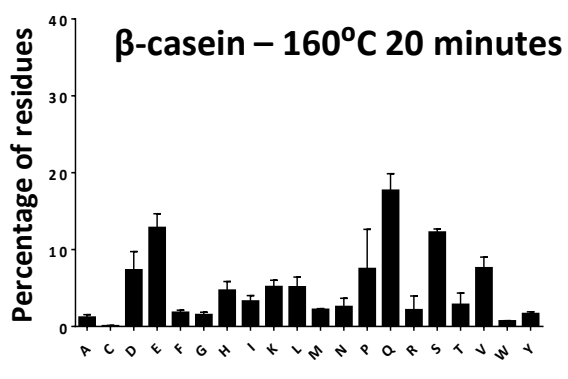

Amino acid residue

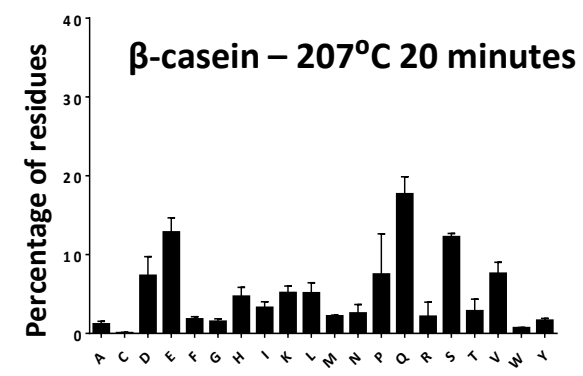

Amino acid residue 


\section{Supplementary Figure 5A}

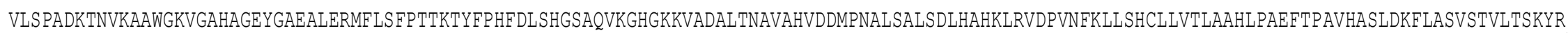
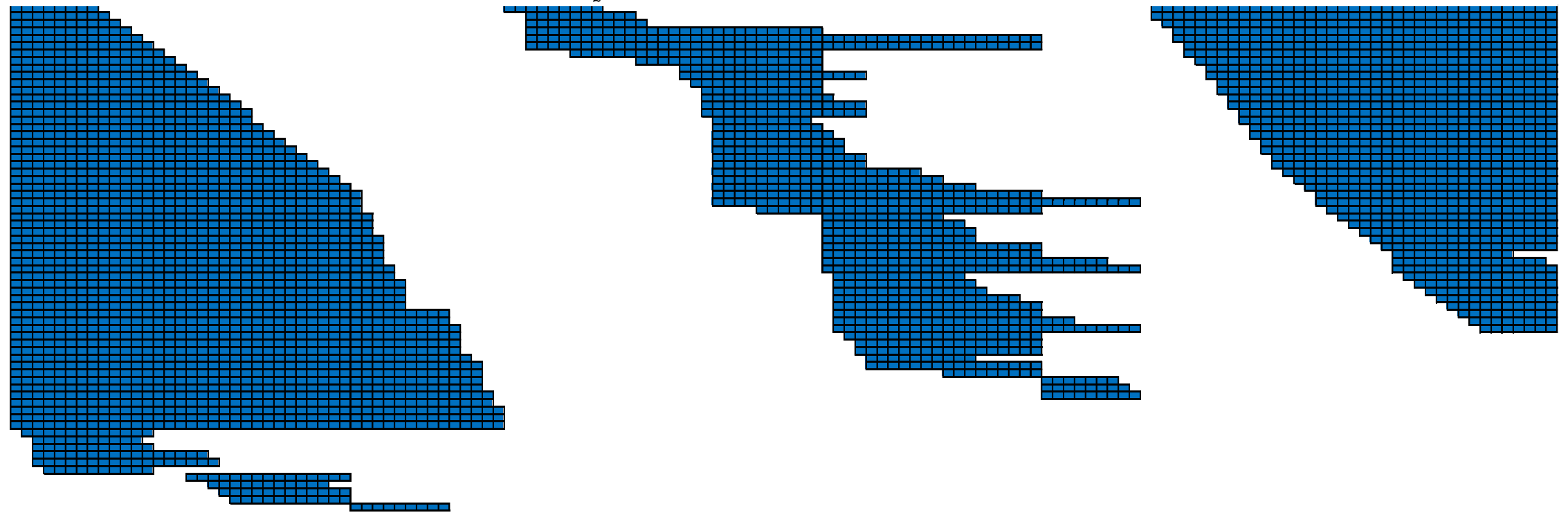

SCW hydrolysis of $\alpha$-globin at $160^{\circ} \mathrm{C}$ for 0 minutes 
VLSPADKTNVKAAWGKVGAHAGEYGAEALERMF LSFPTTKTYFPHFDLSHGSAQVKGHGKKVADALTNAVAHVDDMPNALSALSDLHAHKLRVDPVNNFKLLSHCLLVTLAAHLPAEFTPAVHASLDKKFLASVSTVLTSKYR
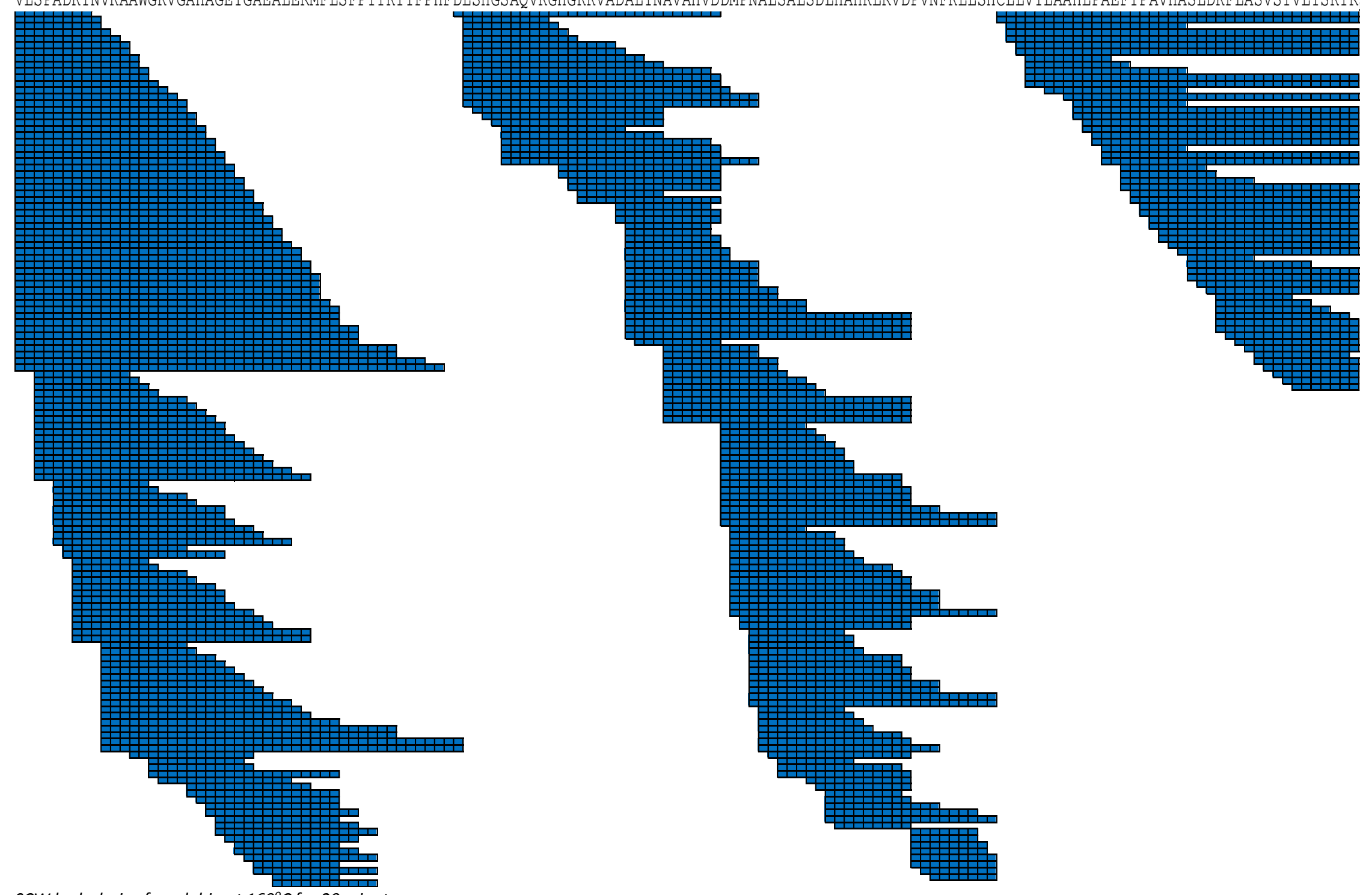
VLSPADKTNVKAAWGKVGAHAGEYGAEALERMF LSFPTTKTYFPHFDLSHGSAQVKGHGKKVADALTNAVAHVDDMPNALSALSDLHAHKLRVDPVNFKLLSHCLLVTLAAHLPAEFTPAVHASLDDKFLASVSTVLTSKYR

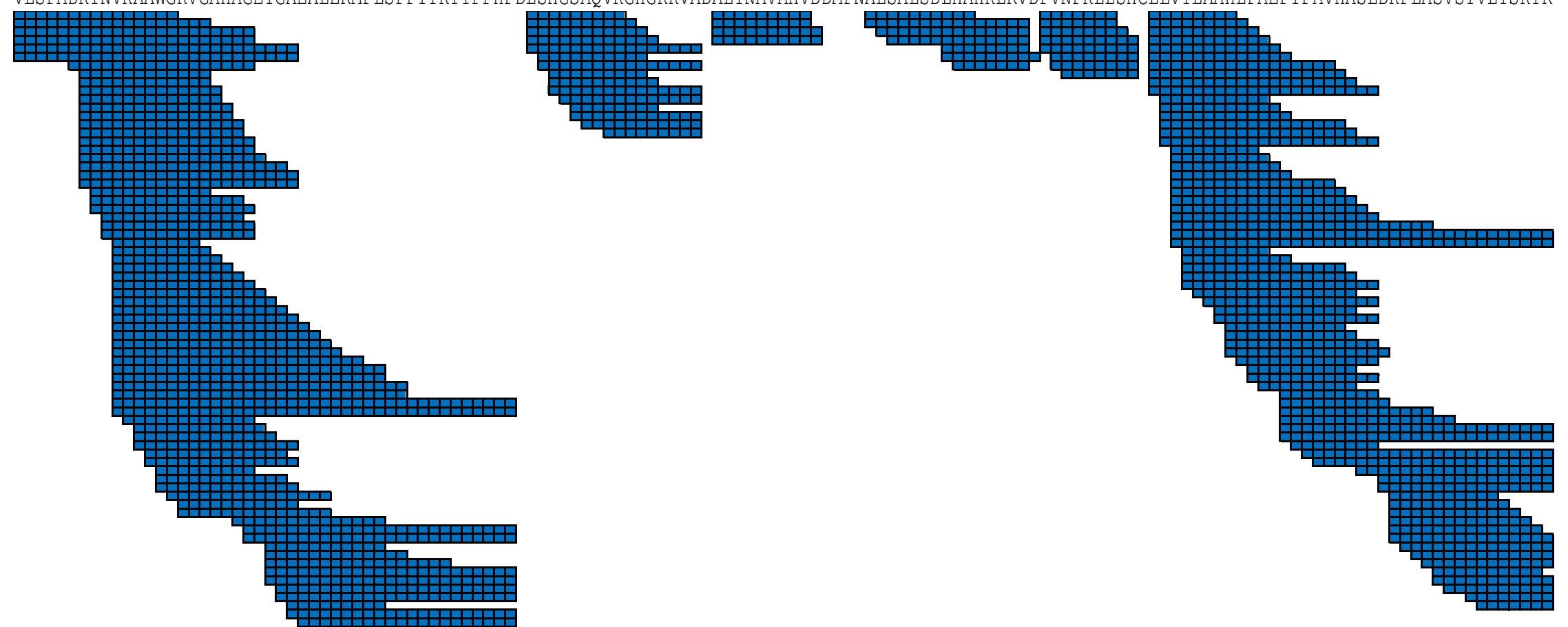

SCW hydrolysis of $\alpha$-globin at $207^{\circ} \mathrm{C}$ for 20 minutes 


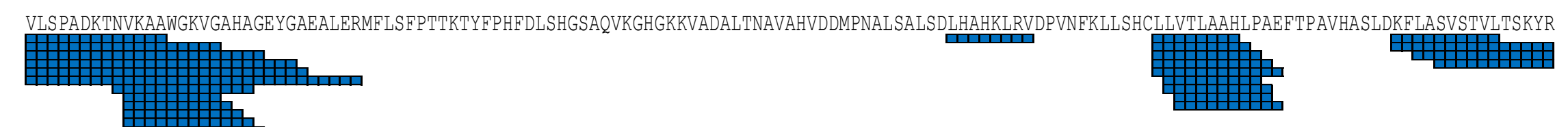

SCW hydrolysis of $\alpha$-globin at $253^{\circ} \mathrm{C}$ for 20 minutes 
VLSPADKTNVKAAWGKVGAHAGEYGAEALERMFLSFPTTKTYFPHFDLSHGSAQVKGHGKKVADALTNAVAHVDDMPNALSALSDLHAHKLRVDPVNFKLLSHCLLVTLAAHL PAEFTPAVHASLDKFLASVSTVLTSKYR SCW hydrolysis of $\alpha$-globin at $300^{\circ} \mathrm{C}$ for 20 minutes 
VLS PADKTNVKAAWGKVGAHAGEYGAEALERMFLSFPTTKTYFPHFDLSHGSAQVKGHGKKVADALTNAVAHVDDMPNALSALSDLHAHKLRVDPVNFKLLSHCLLVTLAAHLPAEFTPAVHASLDKFLASVSTVLTSKYR

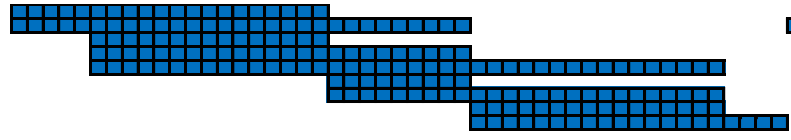

מח

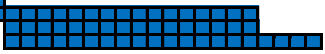

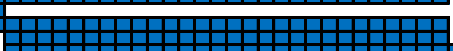

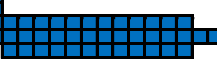

Trypsin digest of $\alpha$-globin 
VHLTPEEKSAVTALWGKVNVDEVGGEALGRLLVVY PWTQRFFESFGDLSTPDAVMGNPKVKAHGKKVLGAFS DGLAHLDNLKGTFATLSELHCDKLHVDPENFRLLGNVLVCVLAHHFGKEFFTPPVQAAYQKVVAGVVANALAHKYH
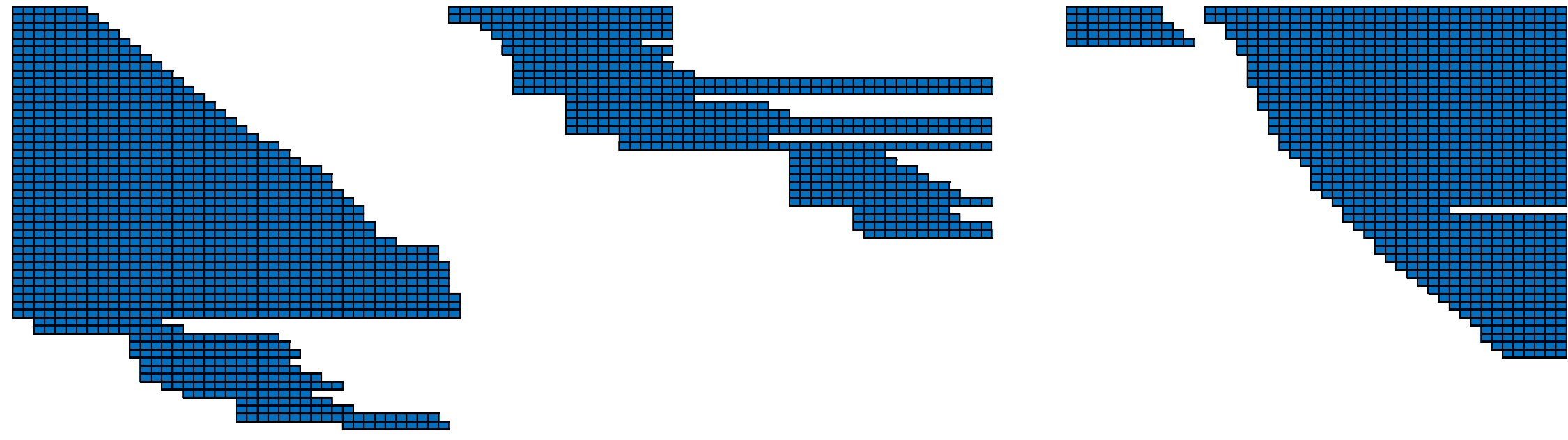

SCW hydrolysis of 6 - globin at $160^{\circ} \mathrm{C}$ for 0 minutes 
VHLTPEEKSAVTALWGKVNVDEVGGEALGRLLVVYPWTORF EESFGDLSTPDAVMGNPKVKAHGKKVLGAFSDGLAHLDNLKGTFATLSELHCDKLHVDPENFRLLGNVLVCVLAHHFGKEFTPPVQAAYQKVVAGVANALAHKYH
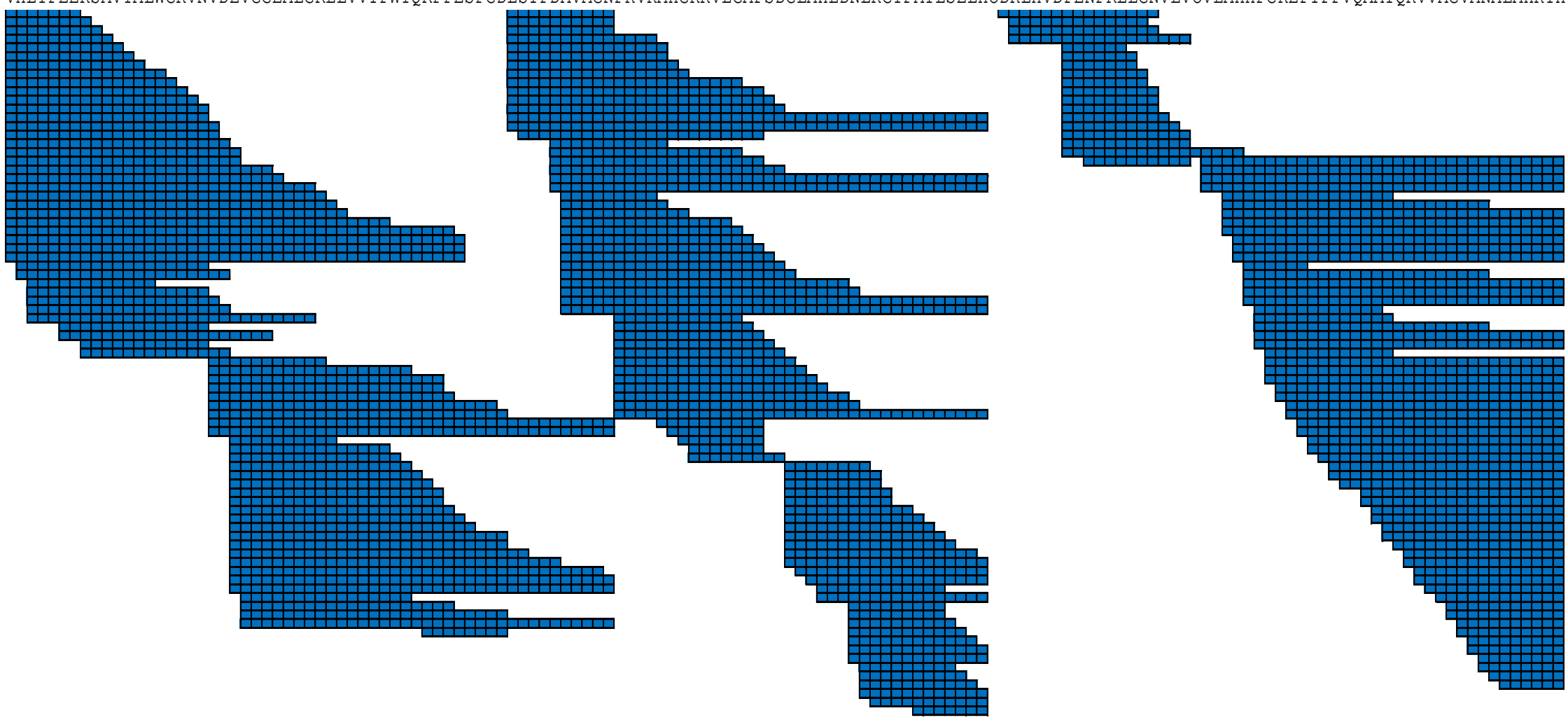

SCW hydrolysis of 6 - globin at $160^{\circ} \mathrm{C}$ for 20 minutes 
VHLTPEEKSAVTALWGKVNVDEVGGEALGRLLVVYPWTQRFFESFGDLSTPDAVMGNPKVKAHGKKVLGAFSDGLAHLDNLKGTFATLSELHCDKLHVDPENFRLIGNVLVCVLAHHFGKEFTPPVQAAYQKVVAGVANALAHKYH
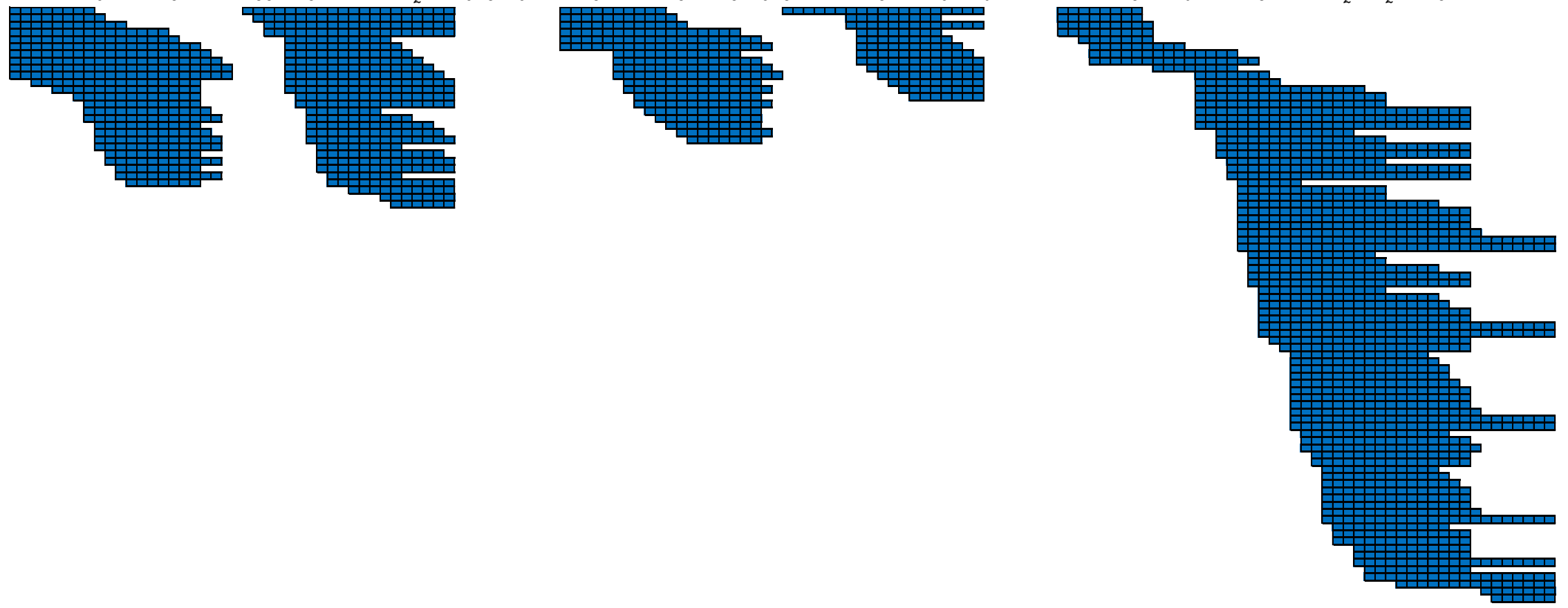

SCW hydrolysis of B-globin at $207^{\circ} \mathrm{C}$ for 20 minutes 
VHLTPEEKSAVTALWGKVNVDEVGGEALGRLLVVYPWTQRFFESFGDLSTPDAVMGNPKVKAHGKKVLGAFS DGLAHLDNLKGTFATLSELHCDKLHVDPENFRLLGNVLVCVLAHHFGKEFTPPVQAAYQKVVAGVANALAHKYH

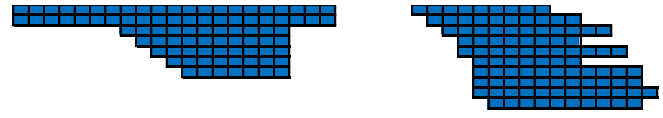

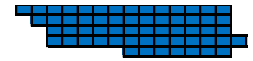

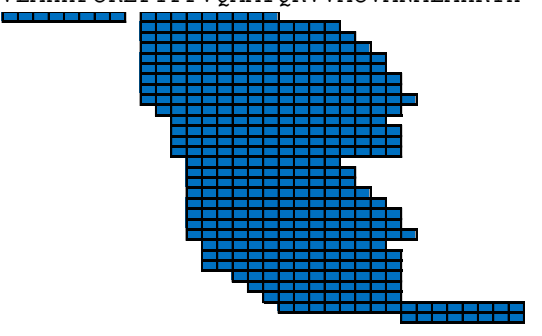

SCW hydrolysis of B-globin at $253^{\circ} \mathrm{C}$ for 20 minutes 
VHLTPEEKSAVTALWGKVNVDEVGGEALGRLLVYYPWTQRFFES FGDLSTPDAVMGNPKVKAHGKKVLGAFS DGLAHLDNLKGTFATLSELHCDKLHVDPENFRLLGNVLVCVLAHHFGKEFTPPVQAAYQKVVAGVANALAHKYH SCW hydrolysis of 6 - globin at $300^{\circ} \mathrm{C}$ for 0 minutes 
VHLTPEEKSAVTALWGKVNVDEVGGEALGRLLVVYPWTORFFESFGDLSTPDAVMGNPKVKAHGKKVLGAFSDGLAHLDNLKGTFATLSELHCDKLHVDPENFRLLGNVLVCVLAHHFGKEFTPPVOAAYOKVVAGVANALAHKYH

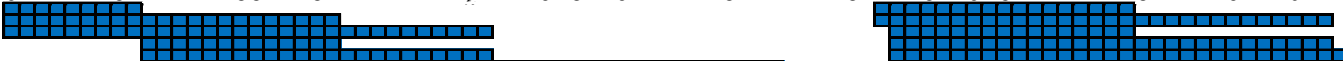

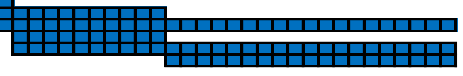

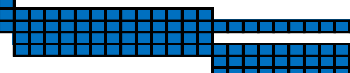

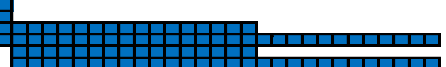

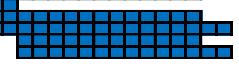

Trypsin digest of B- globin 


\section{Supplementary Figure 5B}

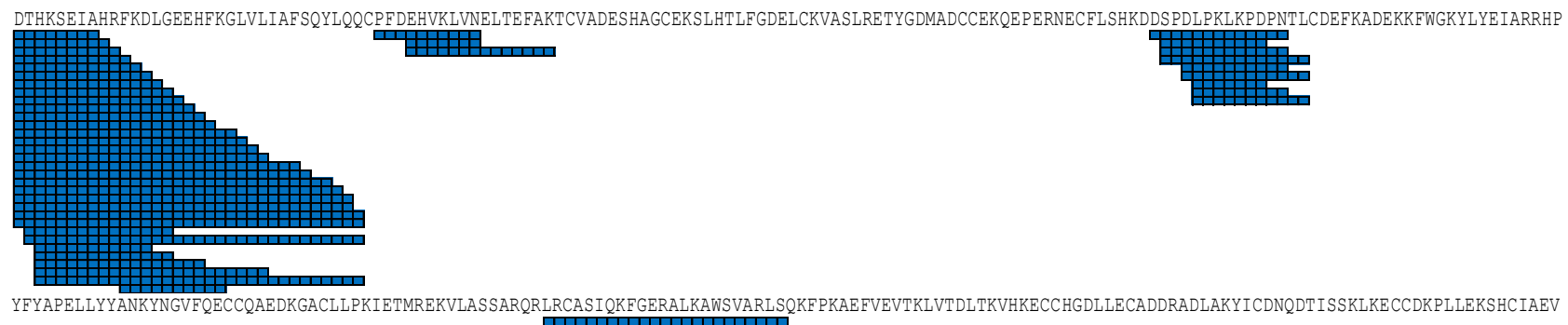

YFYAPELLYYANKYNGVFQECCQAEDKGACLIPKIETMREKVLASSARQRLRCASIQKFGERALKAWSVARLSQKEPKAEFVEVTKI

EKDAIPENLPPLTADFAEDKDVCKNYQEAKDAELGSELYEYSRRHPEYAVSVLLRLAKEYEATLEECCAKDDPHACYSTVFDKLKHLVDEPQNLIKQNCDQEEKLGEYGFQNALIVRYTRKVPPQVSTPTLVEVSRSSLGKVGTRCCT 玵IIIIIIIIII
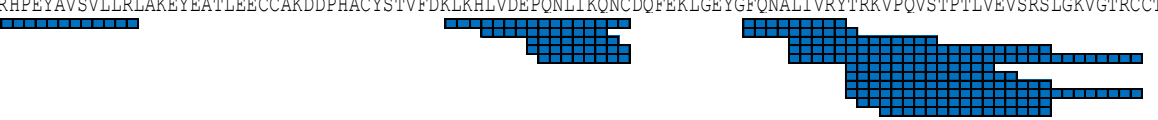

KPESERMPCTEDYLSLILNRLCVLHEKTPVSEKVTKCCTESLVNRRPCFSALTPDETYVPKAFDEKLFTFHADICTLPDTEKQIKKQTALVELLKHKPKATEEQLKTVMENFVAFVDKCCAADDKEACFAVEGPKLVVSTQTALA

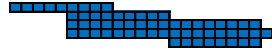

(1)

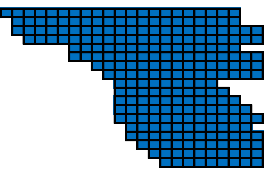



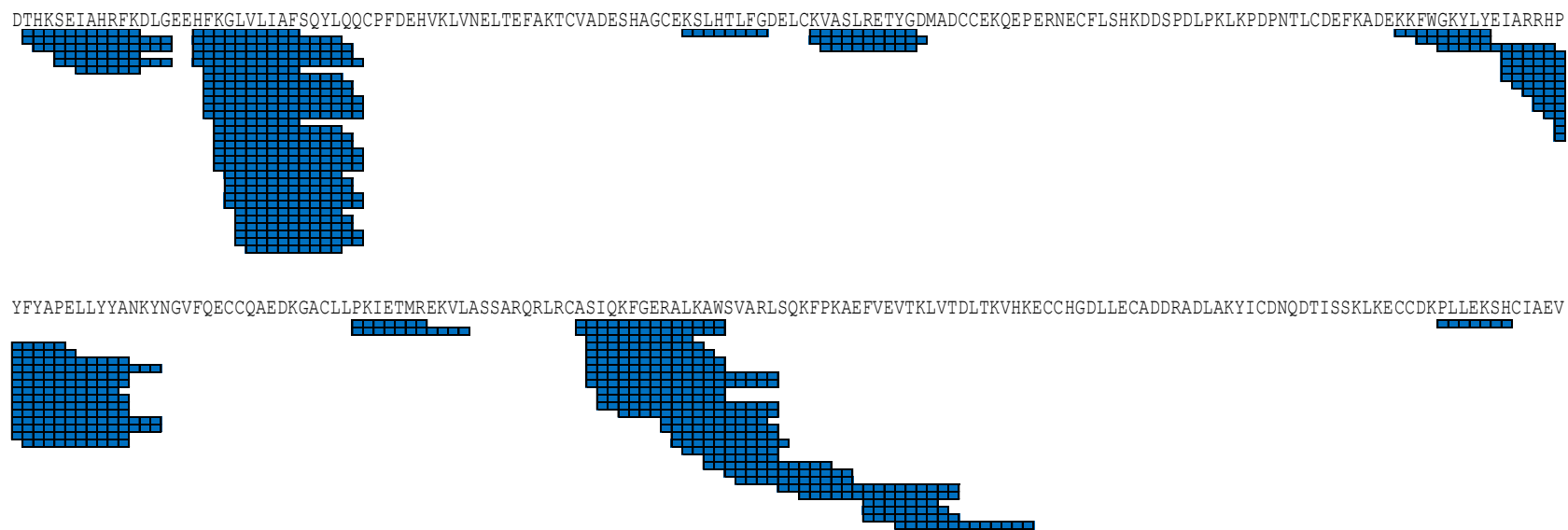

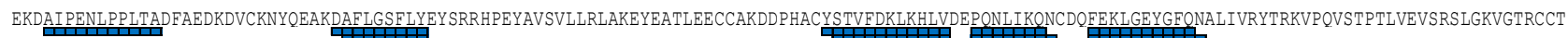
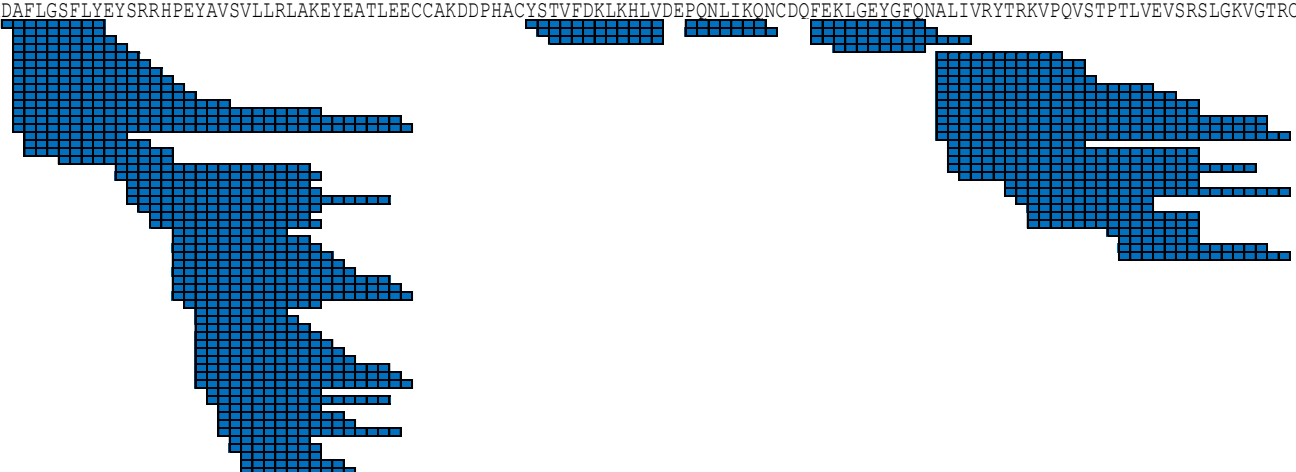

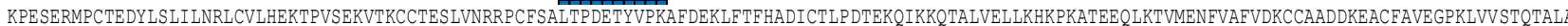
4\#
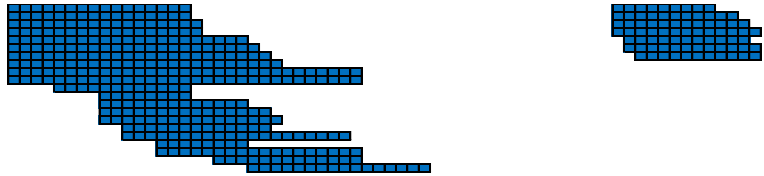

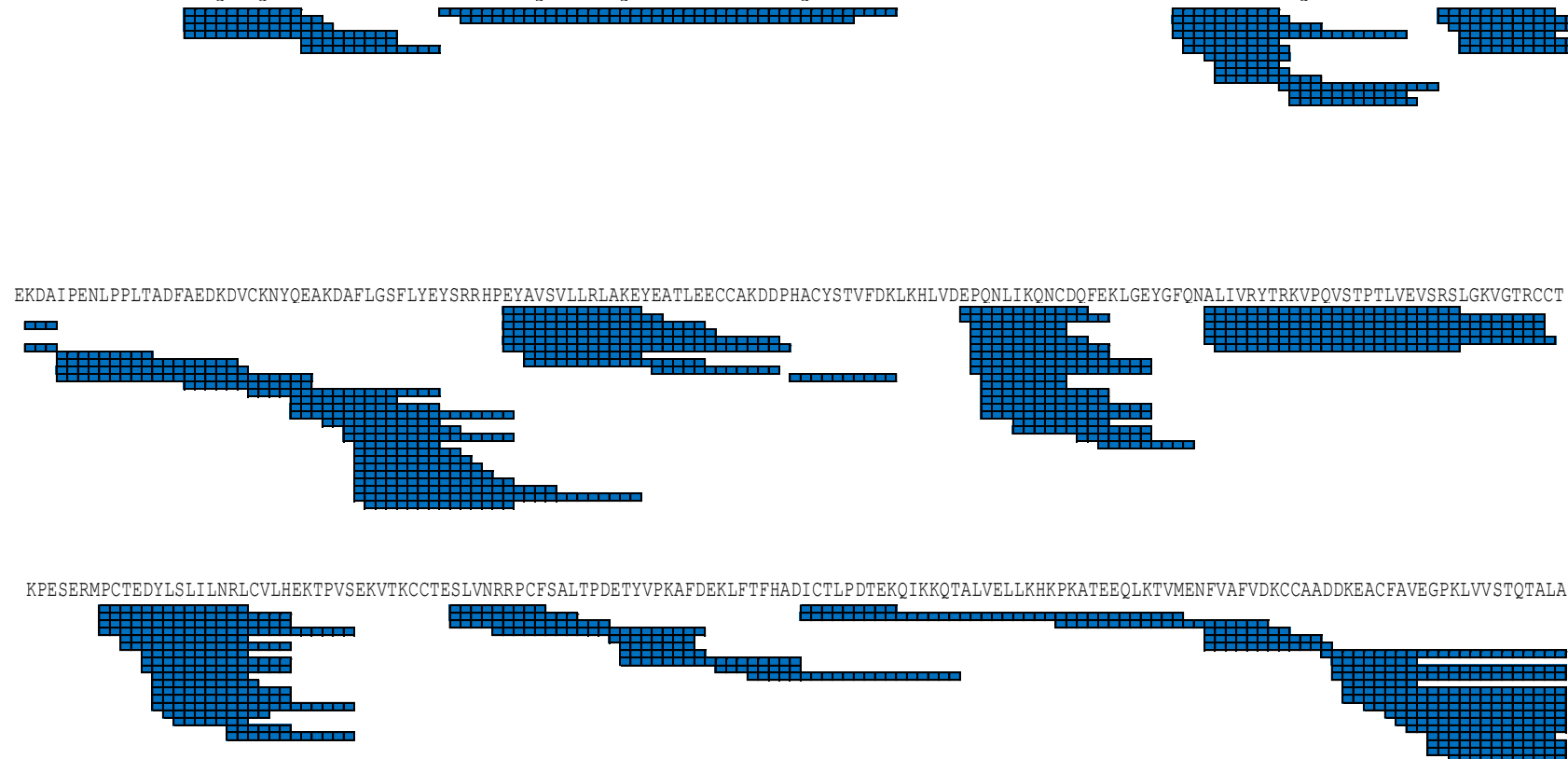

$B S A$ reduction pre- SCW hydrolysis at $160^{\circ} \mathrm{C}$ for 0 minutes 

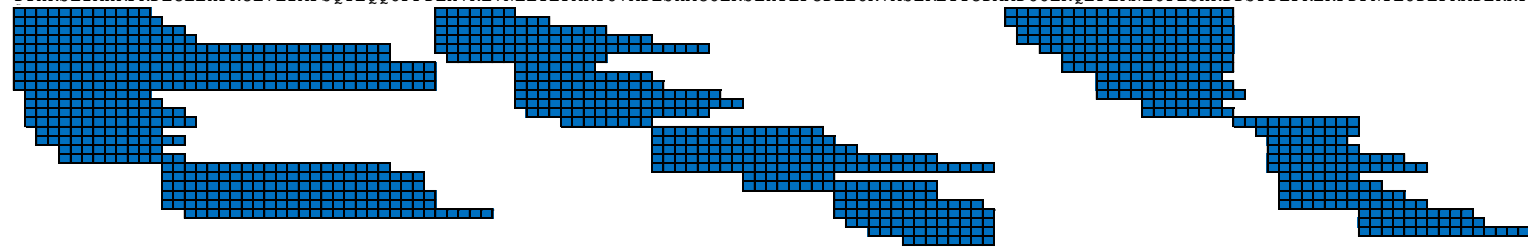

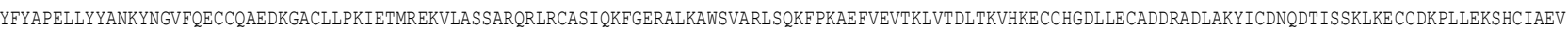
प्म

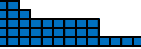

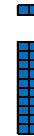

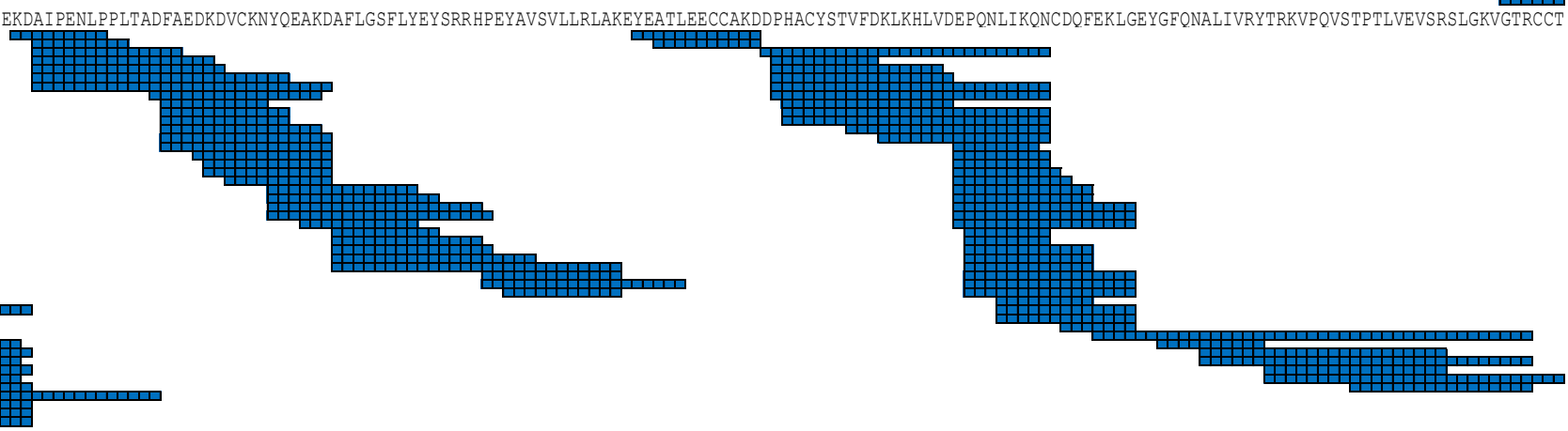

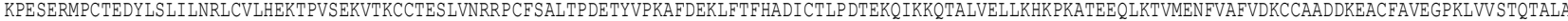


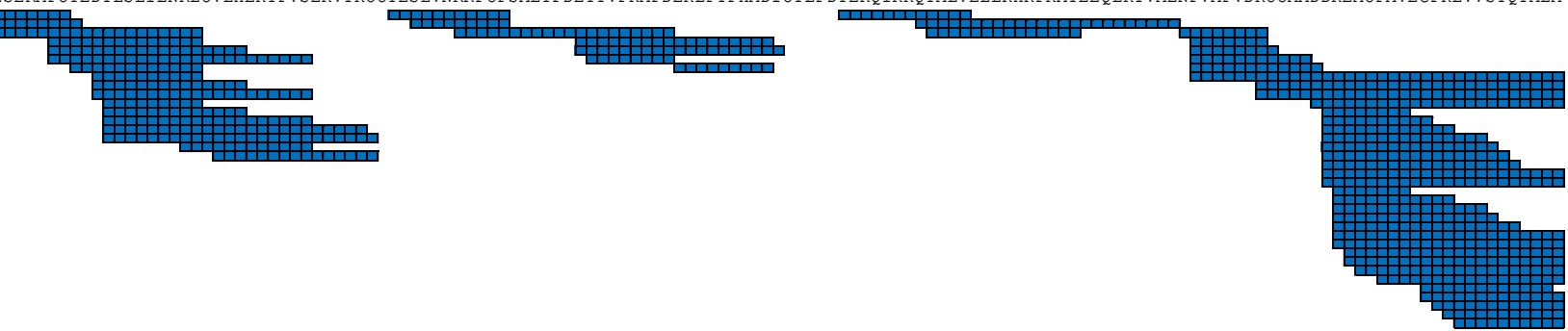

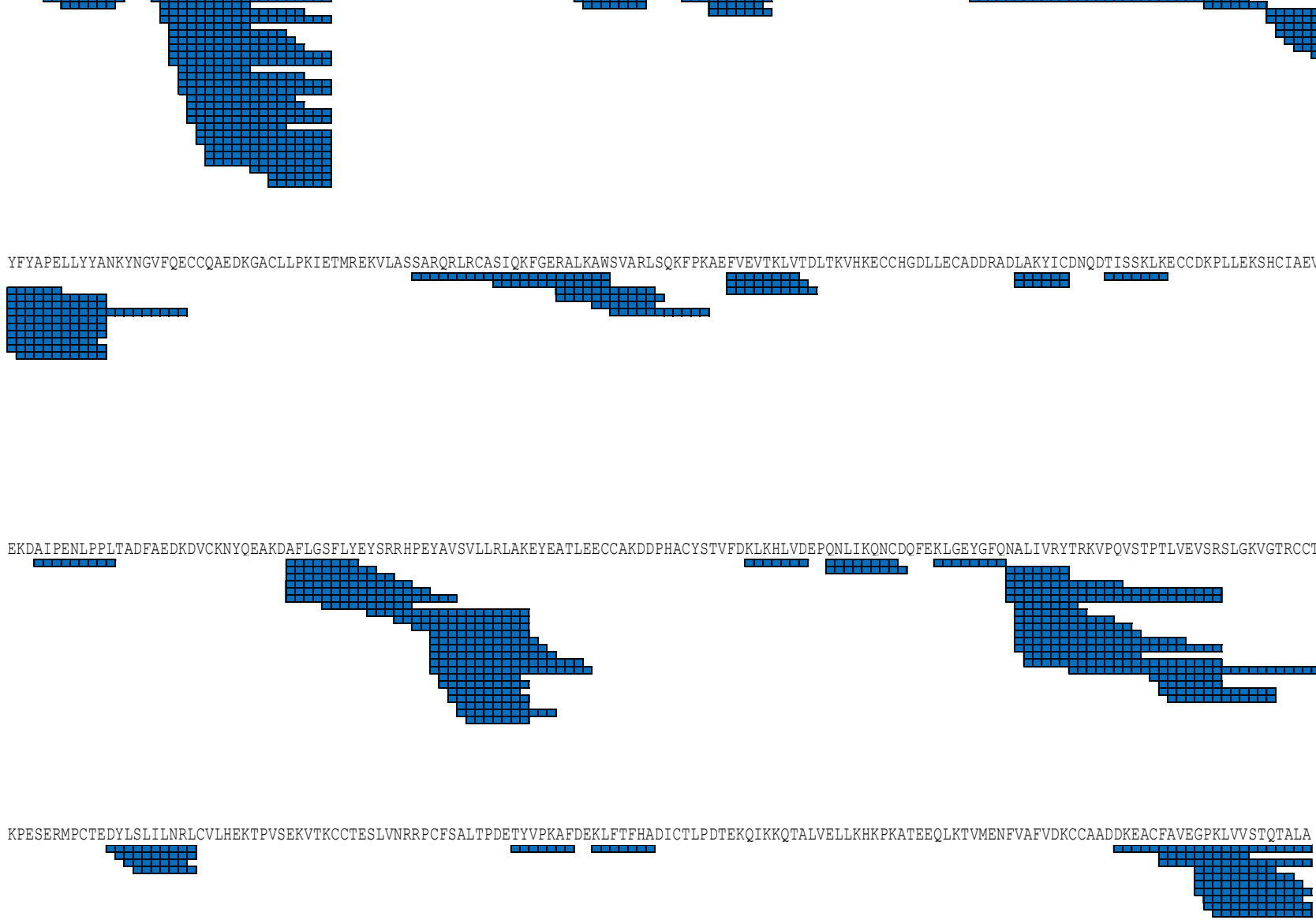


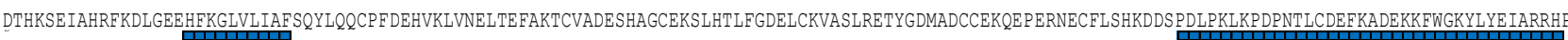
प田腘

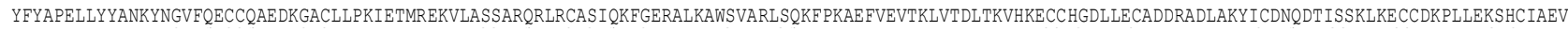

미

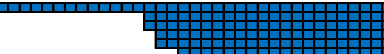

BSA reduction pre- SCW hydrolysis at $253^{\circ} \mathrm{C}$ for 20 minutes 
DTHKSEIAHRFKDLGEEHFKGLVLIAFSQYLQQCPFDEHVKLVNELTEFAKTCVADESHAGCEKSLHTLFGDELCKVASLRETYGDMADCCEKQEPERNECFLSHKDDSPDDLPKLKPDPNTLCDEFKADEKKFWGKYLYEIARRHP

YFYAPELLYYANKYNGVFQECCQAEDKGACLLPKIETMREKVLASSARQRLRCASIQKFGERALKAWSVARLSQKFPKAEFVEVTKLVTDLTKVHKECCHGDLLECADDRADLAKYICDNQDTISSKLKECCDKKPLIEKSHC IAEV

EKDAI PENLPPLTADFAEDKDVCKNYQEAKDAFLGSFLYEYSRRHPEYAVSVLLRLAKEYEATLEECCAKDDPHACYSTVFDKLKHLVDEPQNLIKONCDQFEKLGEYGFONALIVRYTRKVPQVSTPTLVEVSRSLGKVGTRCCT

KPESERMPCTEDYLSLILNRLCVLHEKTPVSEKVTKCCTESLVNRRPCFSALTPDETYVPKAFDEKLFTFHADICTLPDTEKQIKKQTALVELLKHKPKATEEQLKTVMENFVAFVDKCCAADDKEACFAVEGPKLVVSTQTALA

BSA reduction pre-SCW hydrolysis at $300^{\circ} \mathrm{C}$ for 20 minutes 

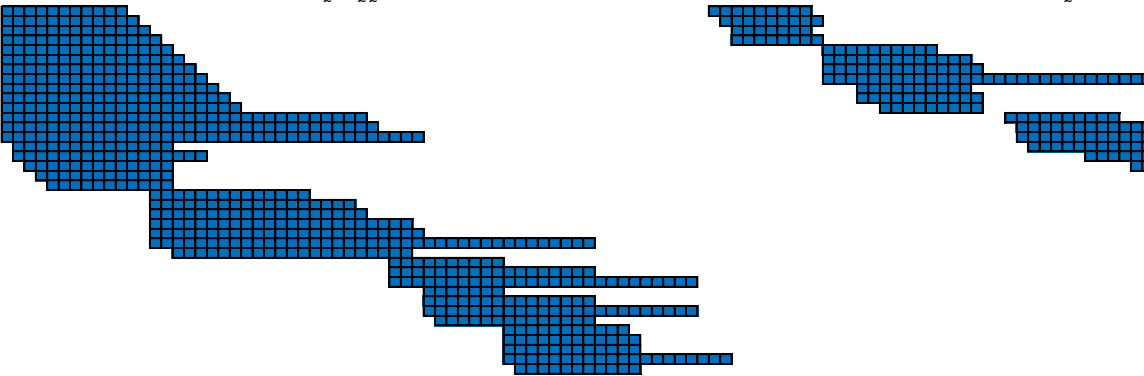

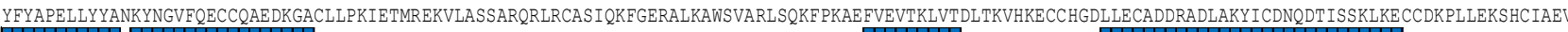

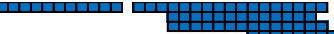

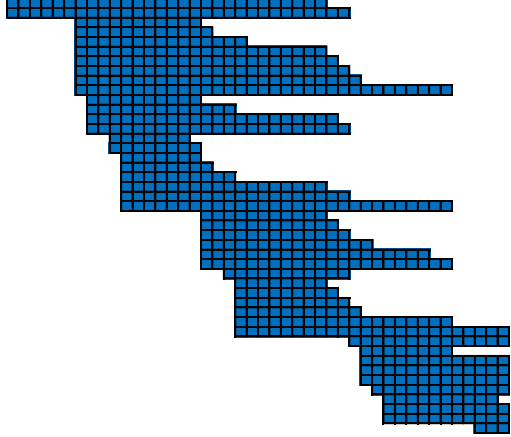

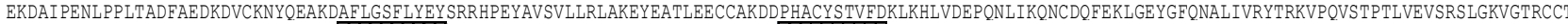
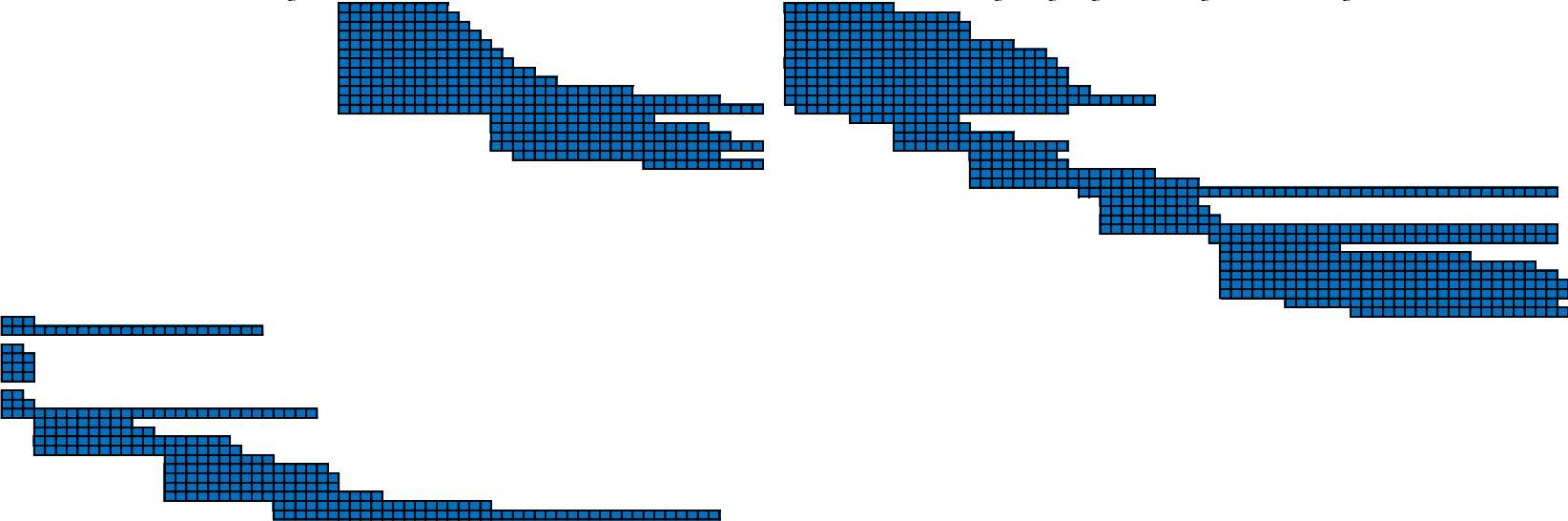

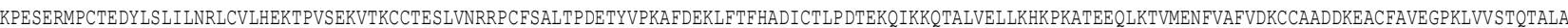
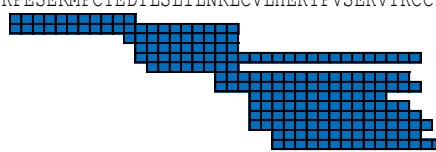


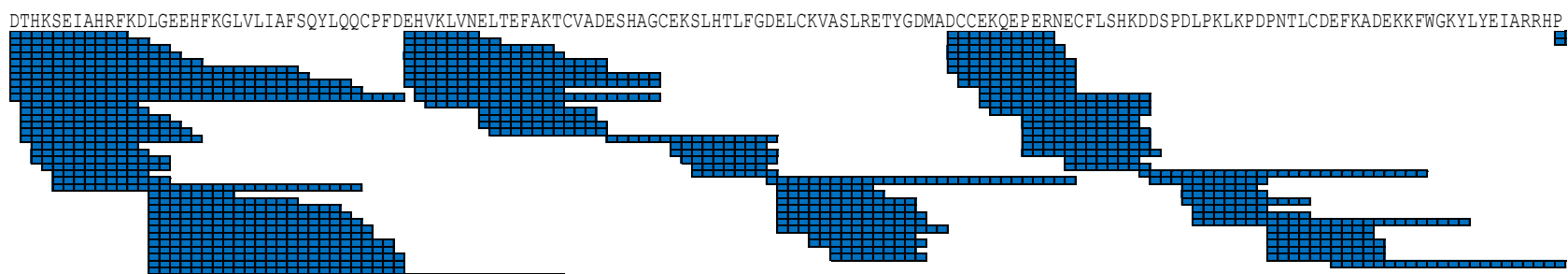

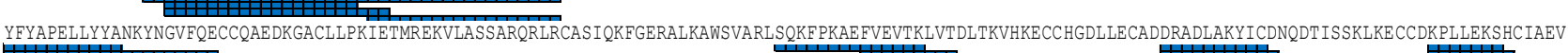
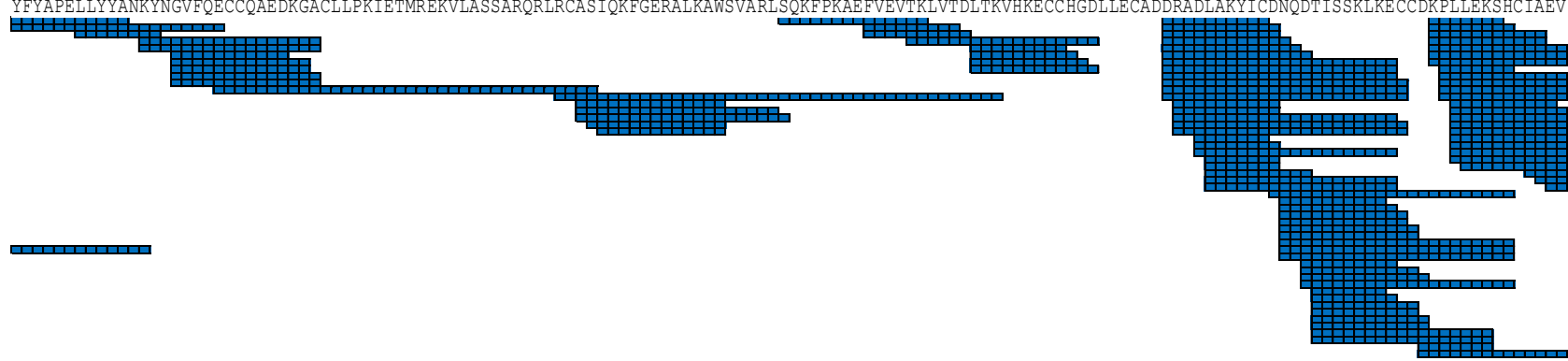

EKDAIPENLPPLTADFAEDKDVCKNYQEAKDAFLGSFLYEYSRRHPEYAVSVLLRLAKEYEATLEECCAKDDPHACYSTVFDKLKHLVDEPQNLIKQNCDQFEKLGEYGFONALIVRYTRKVPQVSTPTLVEVSRSLGKVGTRCCT
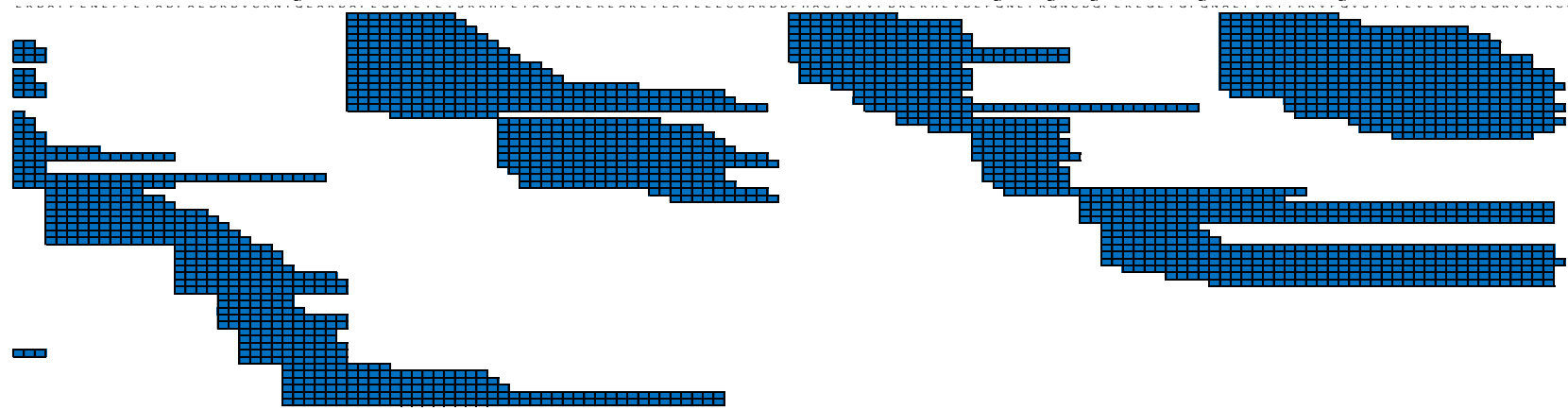

KPESERMPCTEDYLSLILNRLCVLHEKTPVSEKVTKCCTESLVNRRPCFSALTPDETYVPKAFDEKLFTFHADICTLPDTEKQIKKQTALVELLKHKPKATEEQLKTVMENFVAFVDKCCAADDKEACFAVEGPKLVVSTQTALA

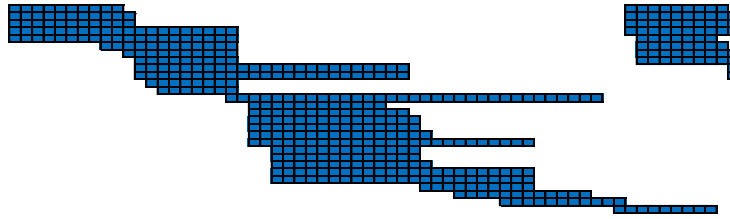

且且且血

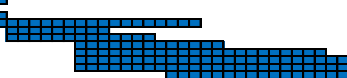

$B S A$ reduction post- SCW hydrolysis at $160^{\circ} \mathrm{C}$ for 20 minutes 

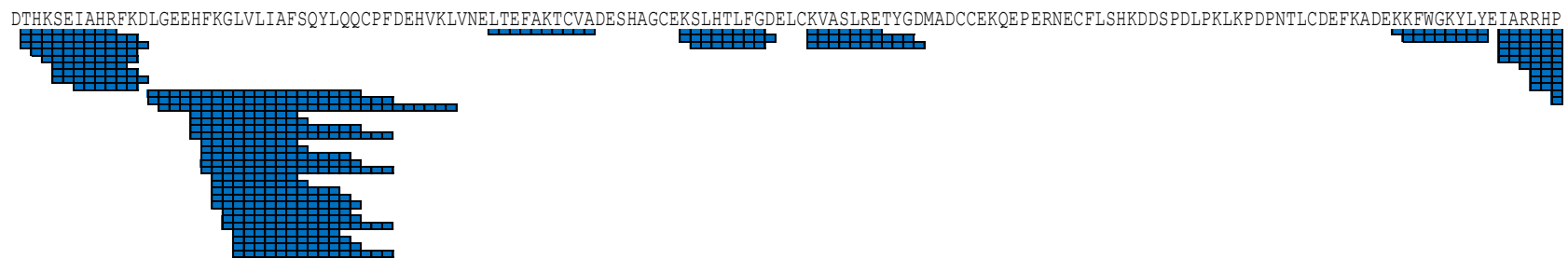

YFYAPELLYYANKYNGVFQECCQAEDKGACLLPKIETMREKVLASSARQRLRCAS IQKFGERALKAWSVARLSQKFPKAEFVEVTKLVTDLTKVHKECCHGDLLECADDRADLAKYICDNQDTTISSKLKECCDKRLLLEKSHC IAEV

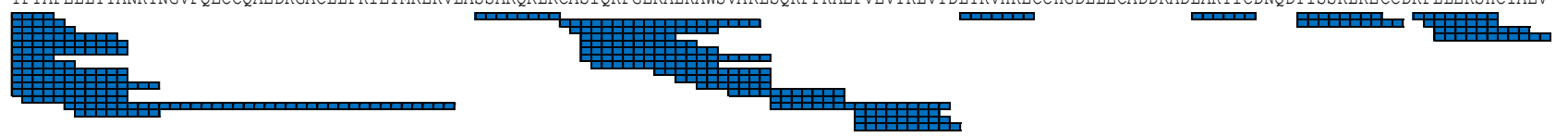

EKDAIPENLPPLTADFAEDKDVCKNYQEAKDAFLGSFLYEYSRRHPEYAVSVLLRLAKEYEATLEECCAKDDPHACYSTVFDKLKHLVDEPQNLIKQNCDQFEKLGEYGEQNALIVRYTRKVPPQVSTPTLVEVSRSLGKVGTRCCT ㅁ 些㰢型

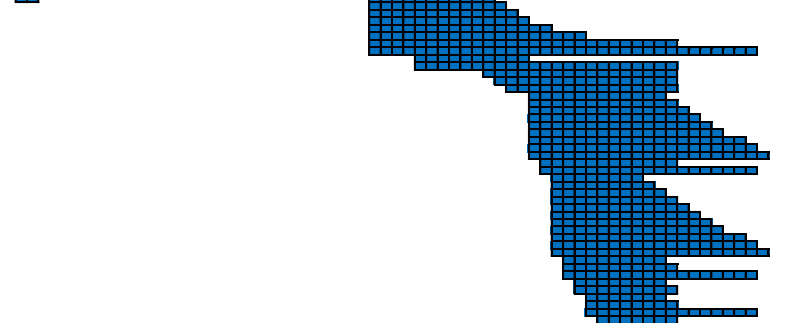

KPESERMPCTEDYLSLILNRLCVLHEKTPVSEKVTKCCTESLVNRRPCFSALTPDETYVPKAFDEKLFTFHADICTLPDTEKQIKKQTALVELLLHKPKATEEQLKTVMENFVAFVDKCCAADDKEACEAVEGPKLVVSTQTALA

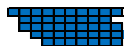

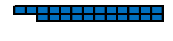

שחוח
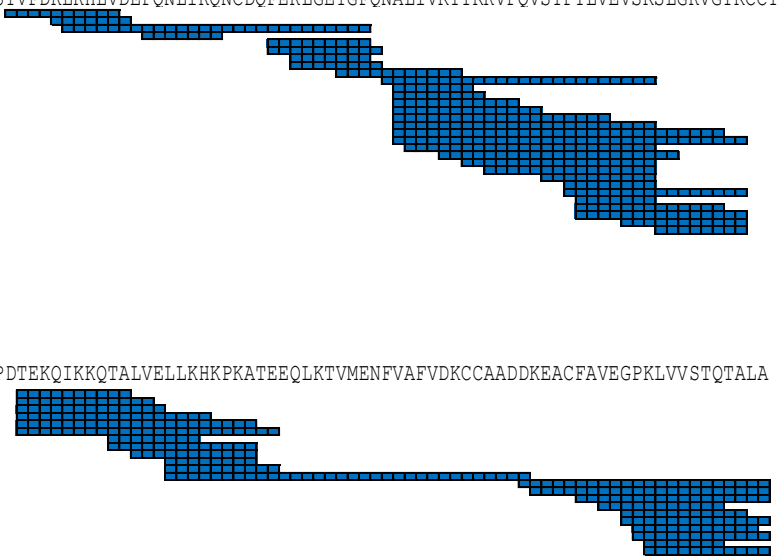

$B S A$ reduction post- SCW hydrolysis at $207^{\circ} \mathrm{C}$ for 20 minutes 


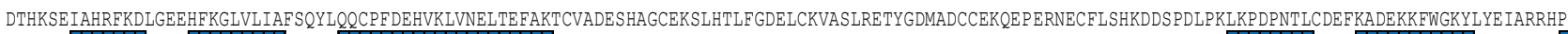
प्य

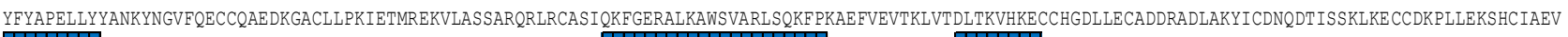

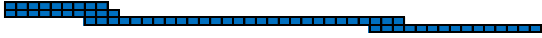

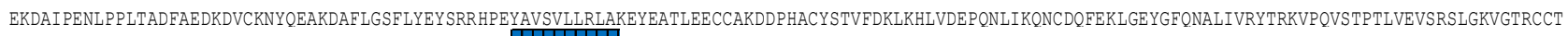

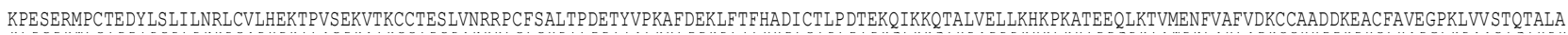
מחד

$B S A$ reduction post- SCW hydrolysis at $253^{\circ} \mathrm{C}$ for 20 minutes 


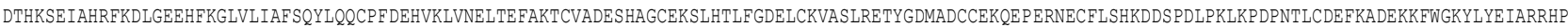

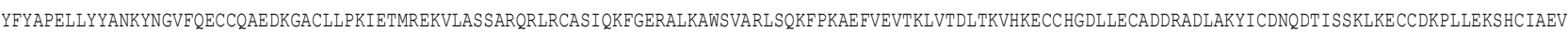

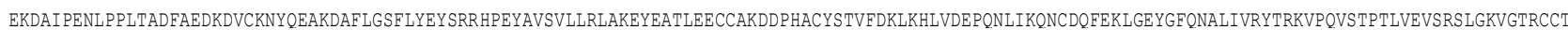

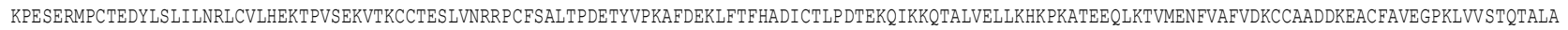

BSA reduction post- SCW hydrolysis at $300^{\circ} \mathrm{C}$ for 20 minutes 


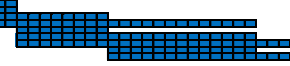




\section{Supplementary Figure 5C}

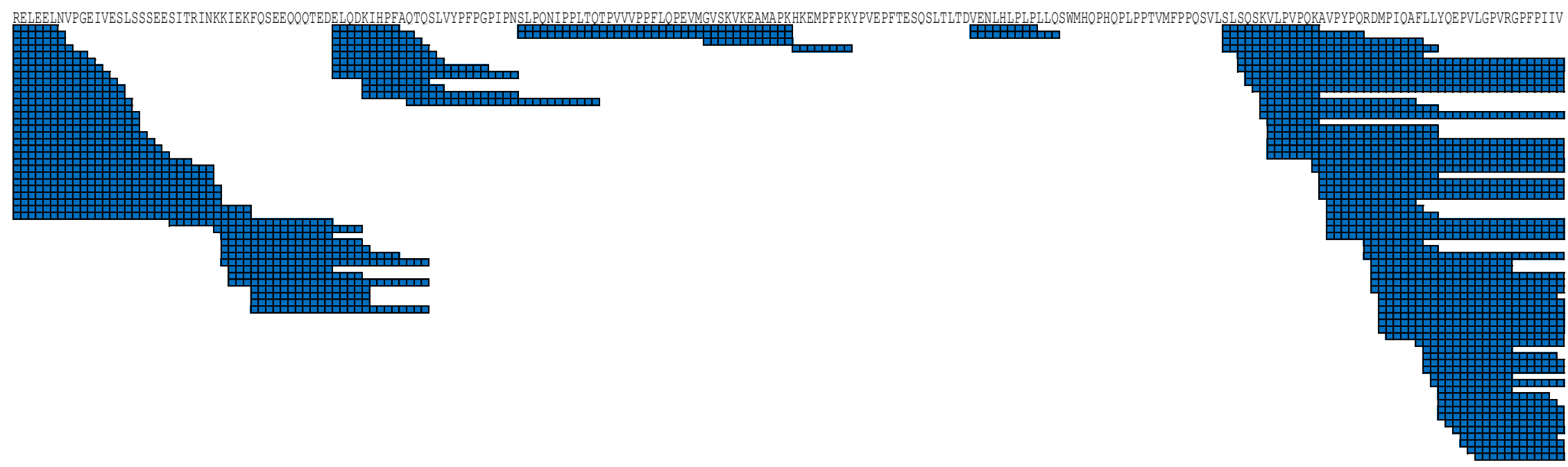

SCW hydrolysis of 8 - casein at $160^{\circ} \mathrm{C}$ for 0 minutes 


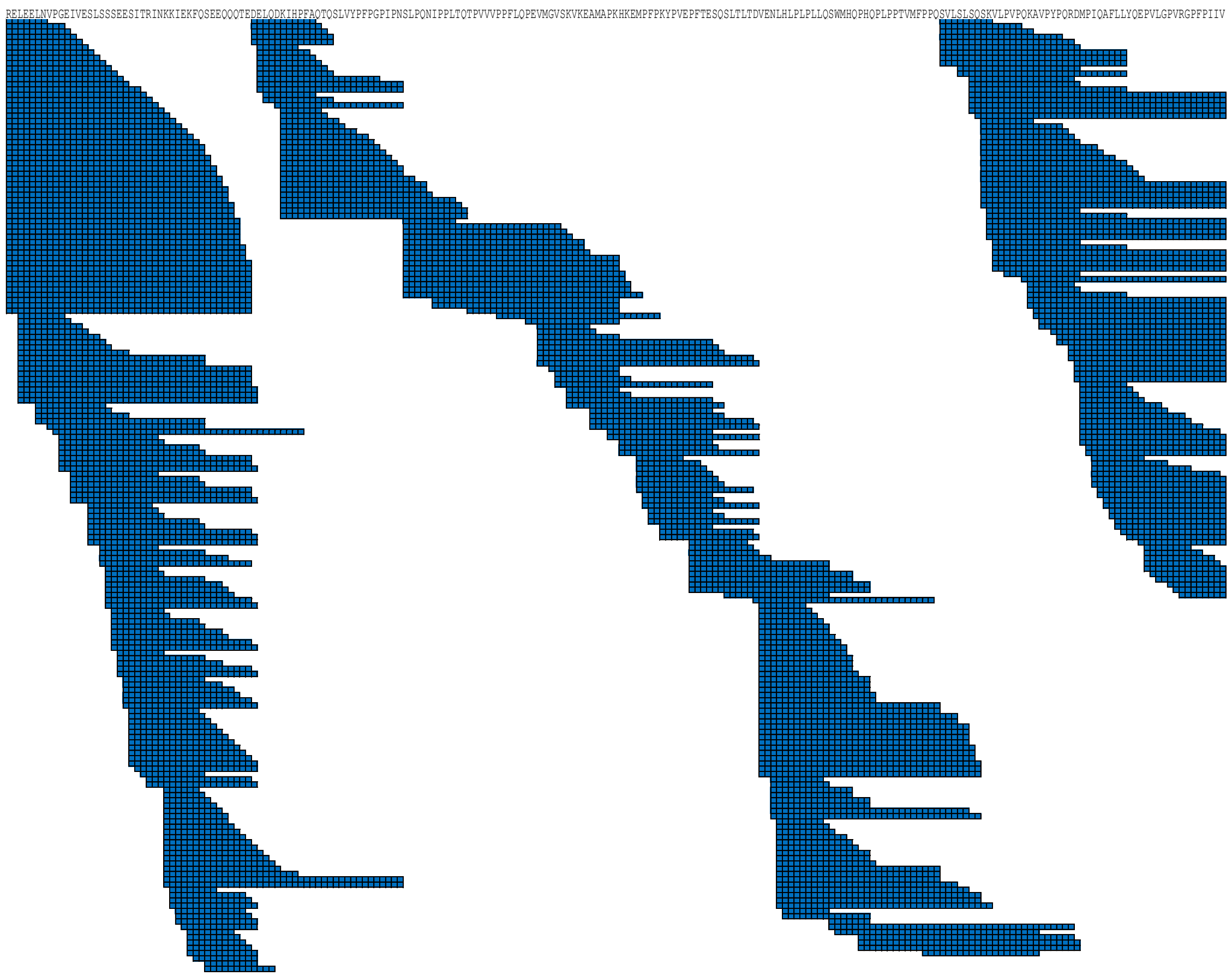




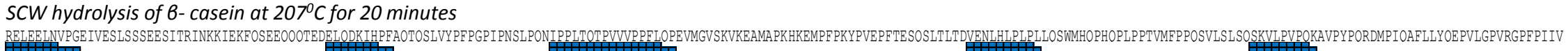

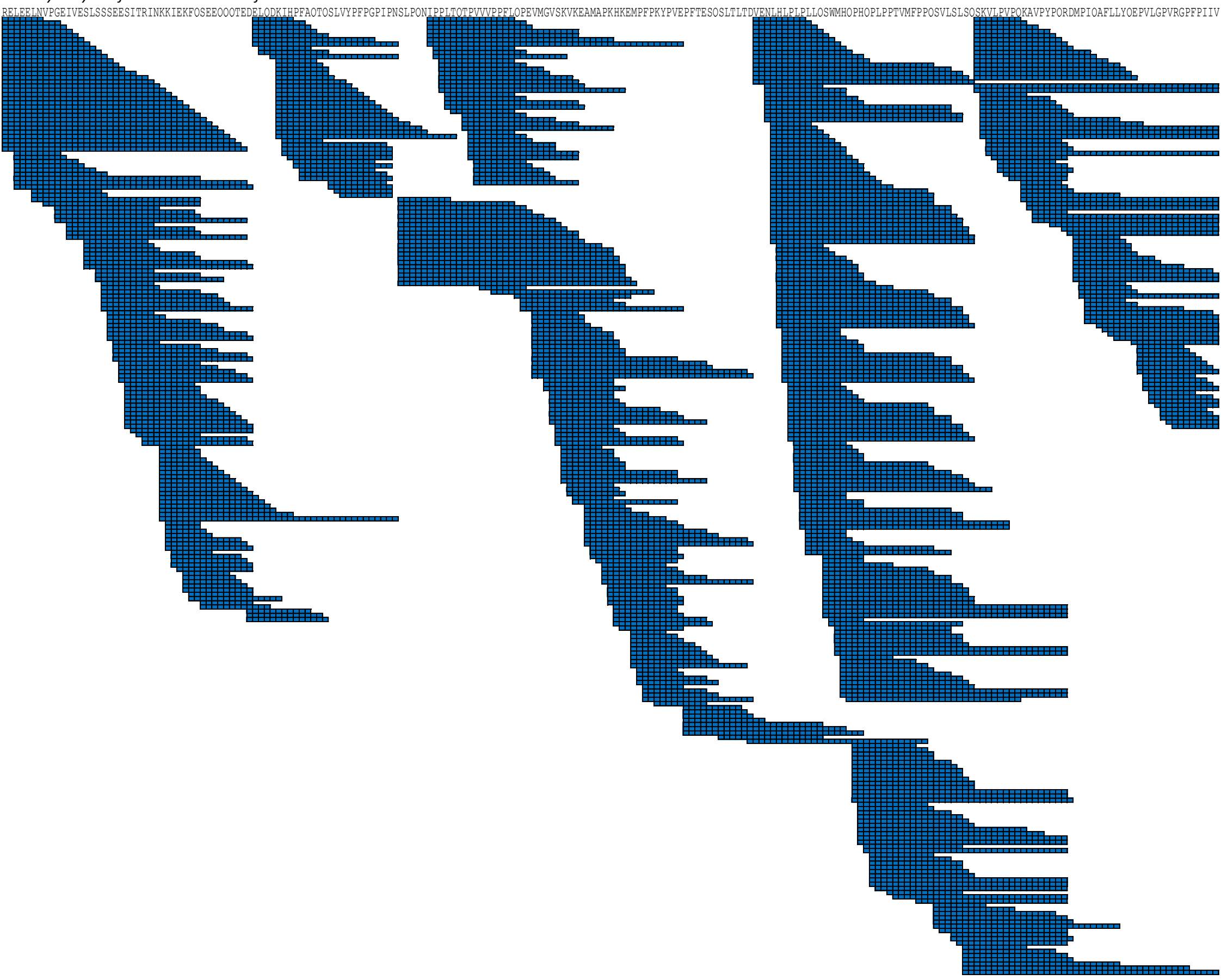




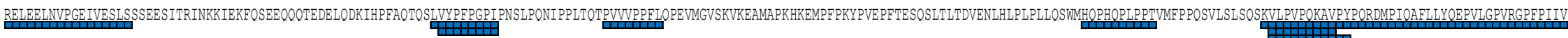

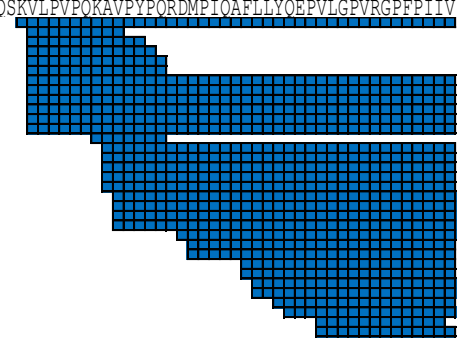

SCW hydrolysis of $\mathrm{B}$ - casein at $253^{\circ} \mathrm{C}$ for 20 minutes 
Supplementary Figure 6 - $\beta$-casein amino acid sequence

RELEELNVPG EIVESLSSSE ESITRINKKI EKFQSEEQQQ TEDELQDKIH PFAQTQSLVY PFPGPIPNSL PQNIPPLTQT PVVVPPELQP EVMGVSKVKE AMAPKHKEMP FPKYPVEPFT ESQSLTLTDV ENLHLPLPLL

QSWMHQPHQP LPPTVMFPPQ SVLSLSQSKV LPVPQKAVPY PQRDMPIQAF LLYQEPVLGP VRGPFPIIV 

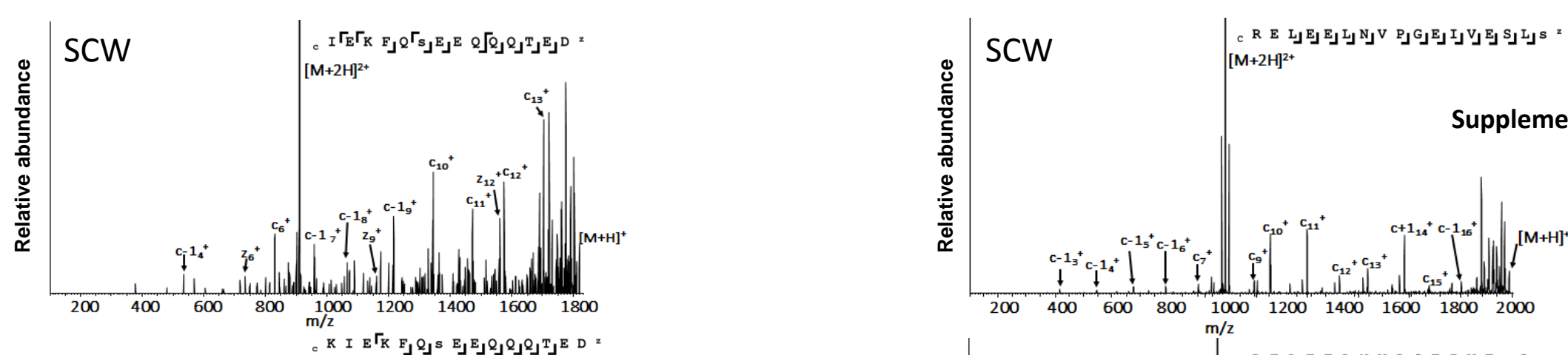

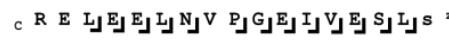

Supplementary Figure 7
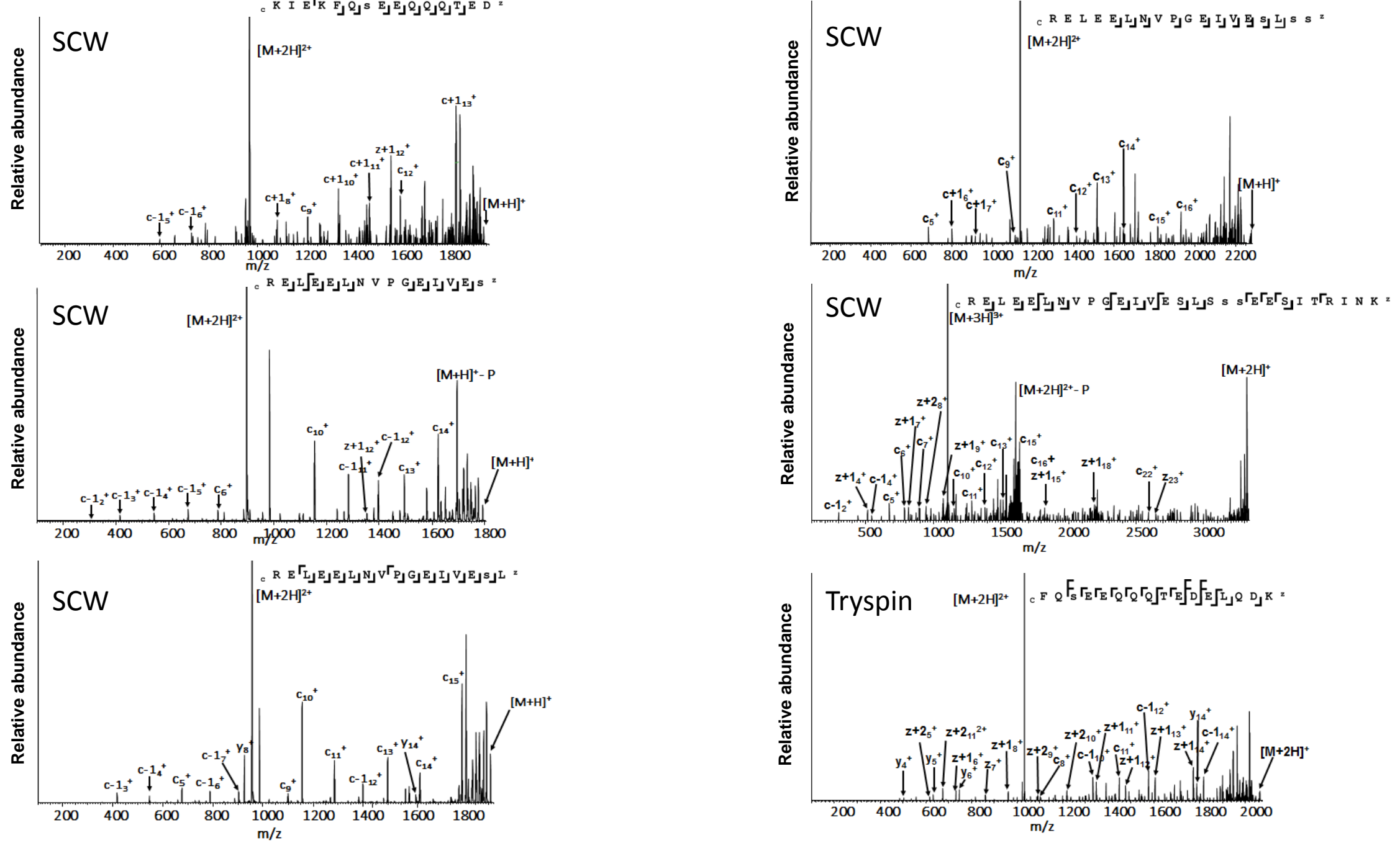